\title{
RECIPROCIDAD HOY: LA RED DE LAS UNIDADES DOMÉSTICAS Y SERVICIOS PÚBLICOS EN DOS COLECTIVOS EN VIC (BARCELONA)*
}

\author{
José Luis MOLINA** \\ Universitat Autonoma de Barcelona \\ Alba ALAYO I GIL *** \\ Universitat Autonoma de Barcelona
}

ABSTRACT: In this paper we analyze examples of reciprocity in a town of $\mathrm{Ca}$ talonia among two collectives related to the Social Welfare Department: aged Catalan people and Berber people immigrated from Nador, Morocco. From a network analysis perspective we propose: a) to differentiate between social exchanges (those exchanges that create social debts, that is, the obligation to reciprocate) and economic and administrative exchanges, balanced in theory, and b) the usefulness of taking the network of domestic units and their exchanges as the unit of analysis. Although the research is in the beginning we have found that reciprocity can be conceptualized as a social exchange naturally attached to domestic sphere but embedded and regulated in the market and Public Administration spheres (as cartel and corruption respectively). To account reciprocity today implies an analysis of the social exchanges in the three domains.

En este articulo analizamos ejemplos de reciprocidad en una ciudad de Catalunya entre dos colectivos relacionados con el Departamento de Bienestar Social: personas de la Tercera Edad y beréberes inmigrados de Nador, Marruecos. Desde una perspectiva de análisis de redes proponemos: a) diferenciar entre intercambios sociales (aquellos intercambios que crean deudas sociales, esto es, la obligación de devolver) e intercambios económicos y administrativos, equilibrados en teoria, y b)

* Esta investigación está financiada por el Proyecto PB98-1238, Programa Sectorial de Promoción General del Conocimiento (MEC). Los nombres usados son seudónimos.

** Divisió d'Antropologia social, Universitat Autònoma de Barcelona. Profesor Ayudante [joseluis.molina@uab.es].

*** Divisió d'Antropologia social, Universitat Autònoma de Barcelona. Estudiante de Doctorado [mariadelalba.alayo@campus.uab.es]. 
la utilidad de tomar la red de unidades domésticas y sus relaciones como unidad de análisis. $A$ pesar de que esta investigación se encuentra en sus inicios proponemos que la reciprocidad puede ser conceptualizada como un intercambio social naturalmente sujeto a la esfera doméstica pero encastado y regulado en la esfera del mercado y de la administración pública (como cartel y corrupción respectivamente). Estudiar la reciprocidad hoy en dia implica un análisis de los intercambios sociales en estos tres dominios.

Cuando pensamos en reciprocidad sabemos que estamos hablando de una característica humana omnipresente, algo que es común a todas las sociedades conocidas. Al igual que otros conceptos fundamentales tomados de las Ciencias Sociales (cultura, religión, etc.), los cuales han sido tratados en profundidad por algunos de los cientificos más importantes, es difícil establecer una única definición del término. Por ello podemos hablar de reciprocidad positiva y negativa, generalizada o equilibrada, capaz de sostener relaciones entre iguales o mantener competiciones entre enemigos (ver Moreno y Narotzky, 2000).

El don sería una forma ceremonial de reciprocidad que incluiría bienes, a menudo con un significado simbólico. Aquí usamos el término reciprocidad como un concepto heurístico (Terradas, 2001) para estudiar aquellos intercambios entre individuos o grupos sociales que conllevan la obligación de beneficiar en el futuro a aquellos que nos han beneficiado primero (o un sentimiento de gratitud si la reparación no es posible) 1 .

Esta amplia noción incluye tres características principales, a saber, la obligación de devolver (i) en el futuro - esto es: no inmediatamente- (ii), los beneficios recibidos de una forma no predeterminada (iii).

No es sorprendente, pues, que intercambios basados en la reciprocidad tengan la tendencia a desarrollar relaciones a largo plazo. Por el contrario, los intercambios orientados por el mercado (como las negociaciones entre vendedores y clientes) o los intercambios de bienes y servicios realizados sobre la base de una norma burocrática (como las multas o la información pública sobre empleos), no implican la obligación de una devolución futura, estando la calidad y cantidad de los bienes y servicios intercambiados en un período dado de tiempo claramente especificadas.

1 Usamos los estudios clásicos en Reciprocidad (MAUSS, 1925; MALINOwSKI, 1922, 1935; POLANYI, 1957; GouldNER, 1960; SAHLINS, 1977 y más recientemente GodELIER, 1996) con nociones extraídas de la teoría del intercambio (HOMANS, 1958; BLAU, 1964; KAPFERER, 1972). 
El papel fundamental de la reciprocidad en las sociedades primitivas ha sido bien establecido desde hace mucho pero tenemos que preguntarnos, ¡cuál es el papel de la reciprocidad en las sociedades complejas? O simplemente, ¿cuál es el papel de la reciprocidad hoy?

Pensamos que existen como mínimo tres argumentos que justifican la realización de esta reflexión.

En primer lugar, se dice (Rifkin, 2000) que el capitalismo está convirtiendo de manera creciente todos los aspectos de la vida social (incluyendo la experiencia humana) en mercancías. Esto podría querer decir que la reciprocidad está retrocediendo en nuestra sociedad. Por esta razón Rifkin (1996) y otros reclaman el refuerzo del papel del Tercer Sector (ONGs, trabajo voluntario y donaciones) en nuestra sociedad.

Segundo, el creciente número de estudios sobre "capital social" (ambos en la formulación original de Bourdieu, 1977, y Coleman, 1988, y en el amplio sentido usado por Putnam, 1993), centrados en el uso instrumental de los recursos sociales para la obtención de ingresos económicos. Los estudios sobre capital social significarían tanto un reconocimiento de la naturaleza encastada de las relaciones económicas (Polanyi, 1944; Granovetter, 1985) como el dominio cada vez más amplio del mercado (y de las relaciones orientadas por el mercado) en la sociedad (para una revisión detallada ver Lin, 2001).

Tercero, y relacionado con las argumentaciones anteriores, la importancia de las redes de apoyo informal de las personas dependientes para las políticas de Bienestar Social (Litwin, 1996:2). La existencia de redes (parientes, vecinos, conocidos) que complementan el sistema formal de asistencia se da por supuesta.

En este artículo analizamos la reciprocidad hoy en una pequeña ciudad situada en el centro de Cataluña (España) desde una perspectiva de análisis de redes. Tomando el Departamento de Bienestar Social del Ayuntamiento como punto de partida hemos identificado dos colectivos, entre otros. El primer colectivo está formado por personas ancianas, residentes en Vic, con una fuerte dependencia de ayuda externa. El segundo colectivo está formado por personas más jóvenes emigradas de Marruecos. En ambos casos hemos identificado las unidades domésticas como unidad de análisis y la red de relaciones que las rodean. Además, hemos establecido una primera red de unidades domésticas, incluyendo las organizaciones públicas con las que están relacionadas. Aunque esta investigación está en sus inicios proponemos la utilidad de considerar las 
unidades domésticas como unidad de análisis y la utilidad de estudiar los intercambios entre ellas para poder obtener una visión del papel de la reciprocidad en nuestra sociedad.

\section{Marco teórico}

Para estudiar los intercambios observados en nuestra investigación proponemos una conceptualización en tres esferas y sus interacciones mutuas: la esfera de las unidades domésticas, la esfera del dominio público (incluyendo ONGs e instituciones religiosas, intimamente relacionadas) y la esfera del mercado. Entre estas tres esferas se dan intercambios continuos. Las unidades domésticas precisan de la esfera pública para servicios como la educación y la salud, pagan impuestos, proporcionan información, etc. Los individuos y las organizaciones que operan en el mercado reciben regulaciones y control y contribuyen con bienes, impuestos y servicios, aunque la recepción de ayuda financiera sea también posible. Finalmente, la esfera de las unidades domésticas intercambia trabajo y capital con la esfera del mercado para obtener bienes, salarios y rentas.

Somos conscientes que esta interpretación puede ser discutida de diferentes maneras, pero pensamos que es útil porque ilumina la naturaleza de las relaciones propias en cada esfera. Gráficamente:

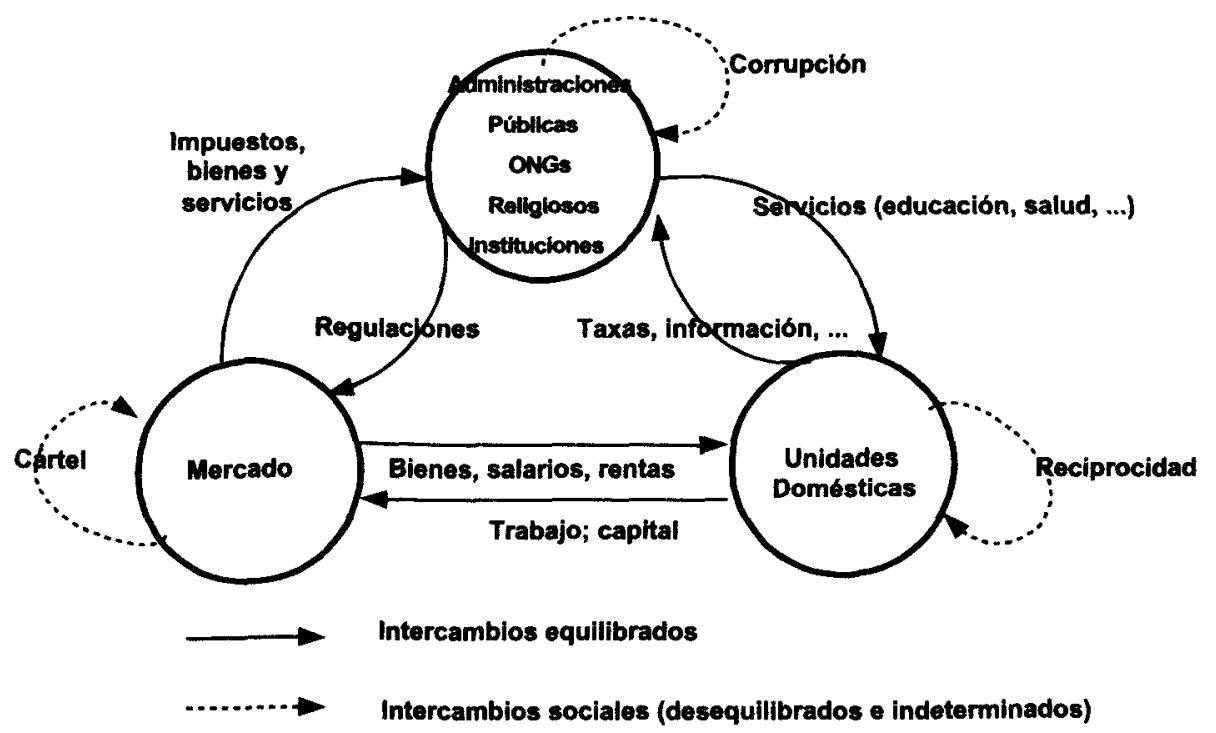


En esta representación, los intercambios entre las unidades domésticas pueden ser conceptualizados como reciprocidad al tratarse de intercambios sociales, o lo que es lo mismo, intercambios entre unidades sociales que implican la obligación de beneficiar en el futuro a aquellos que han beneficiado primero. Estos intercambios pueden ser clasificados (Attias-Donfut y Rozenkier, 1996:39) como socialización (relaciones sociales, relaciones expresivas, pequeños favores), trabajo doméstico (que incluye pequeñas reparaciones y gestiones administrativas) y ayuda financiera (en forma de créditos sin intereses o, simplemente, una transferencia monetaria).

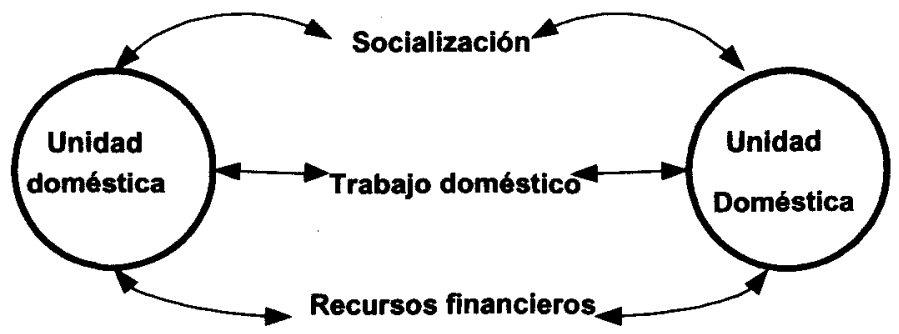

A pesar de que entre las unidades domésticas, al igual que entre individuos, la tendencia de los intercambios tiende al equilibrio, estos intercambios fomentan futuras interacciones y construyen y mantienen relaciones duraderas.

En las otras dos esferas, los intercambios están regidos por el precio (en la esfera de mercado) o por la norma (en la esfera pública) y formalmente está prohibido o al menos desaconsejado introducir obligaciones personales futuras en las operaciones. A través del pago del precio o por el cumplimiento de la norma burocrática, la cual especifica las características del intercambio, las obligaciones futuras desaparecen. En el caso de compromisos futuros, como garantías o hipotecas, éstos son establecidos a través de un contrato.

Si hay intercambios sociales en la esfera de la administración pública más allá de la mera socialización, como información privilegiada, promoción de empleo, recursos financieros y otros, este contrato es visto como corrupto. De la misma manera, si en la esfera de mercado se dan relaciones o asociaciones que tratan de disolver la competencia, entonces hablamos de cartel, monopolio, etc.

Naturalmente, la socialización no puede ser prohibida fuera de la esfera doméstica porque tanto el mercado como la burocracia son constructos sociales encastados en las relaciones sociales, pero se limita de forma estricta las nor- 
mas de etiqueta. No obstante, los intercambios sociales se dan en ambas esferas y los estudios de capital social son un buen indicador de esta presencia. Per ello, sugerimos que el estudio de la reciprocidad en nuestra sociedad tendría que incluir los intercambios sociales que se dan en las tres esferas. Posiblemente la evitación de la incertidumbre en un contexto público de desconfianza (ver la explicación de la mordida mexicana o la palanca chilena en Adler-Lomnitz, 1994) o la falta de regulaciones y control en el mercado pueden explicar la prevalencia en cada caso de intercambios sociales en la esfera de la administración pública o en la esfera del mercado respectivamente.

\section{Reciprocidad en la esfera doméstica}

Vic es una ciudad de $\mathbf{3 0 . 0 0 0}$ habitantes situada en el centro de Cataluña. Esta zona se caracteriza por tener una alta tasa de personas de la Tercera Edad (el $20 \%$ de la población tiene más de 65 años), y en la última década, de un continuo proceso de inmigración básicamente desde la provincia de Nador, una zona rural de las montañas del Rif en el norte de Marruecos, cerca de Melilla (emplazamiento español al norte de África). Éste es un lugar donde, a pesar de la conquista arábiga en siglo VII, la población mantiene aún sus costumbres beréberes mezcladas con las costumbres árabes. Esta población se denomina ella misma imazighen, que significa en su lengua "hombres libres" (beréber es la denominación que les dieron los griegos antiguos) y han mantenido su propia lengua, el tamazight. Hoy en día la proporción de inmigrantes es de alrededor de un 5-6\% (Ajuntament de Vic, 2000). Estos inmigrantes son, en general, hombres con varios años de residencia en Vic, que trabajan básicamente en la industria o en el sector de la construcción y hablan en su mayoría español, y mujeres más jóvenes, con menos años de residencia en general que los hombres, que han llegado después de la regularización obtenida por sus maridos a través de la reagrupación familiar. Muchas de estas mujeres sólo hablan la lengua bereber y actualmente algunas de ellas siguen cursos de alfabetización y catalán.

En los países arabo-musulmanes existe, tradicionalmente, una fuerte separación entre la esfera privada de las mujeres, responsables de todo aquello que concierne el hogar, niños y personas mayores, y la esfera pública, típicamente reservada a los hombres, obligados por el Corán a mantener sus esposas (nafaqa). Este dualización se mantiene en Marruecos y también entre los beréberes de las poblaciones rurales 
de Nador. Sin embargo, algunas mujeres, mayoritariamente de áreas urbanas, están rompiendo actualmente esta separación gracias a una mayor educación y posibilidades de trabajo que les obliga a salir al exterior. Pero, en general, este modelo tradicional de vida continua manteniéndose en Vic después del proceso de inmigración. Muchas de las mujeres no tienen trabajo o no intentan tenerlo, y se quedan en sus casas cuidando a sus hijos y a cargo de las tareas domésticas. Los hombres a través del trabajo mantienen contactos con la población local y aprenden a hablar en catalán o castellano. Los niños (nacidos en Marruecos o en Cataluña) están escolarizados y hablan, con mayor o menor fluidez, catalán y beréber y con menor fluidez, castellano. Estos niños suelen ayudar a las madres y parientes femeninos a comunicarse con médicos, vendedores y la administración pública en general.

Los beréberes adoptaron el Islam como religión después de la conquista arábiga de sus territorios. Actualmente existen dos pequeñas mezquitas en la ciudad en la que sólo acuden los hombres porque son demasiado pequeñas para acomodar un espacio para las mujeres. En Marruecos (y otros países musulmanes) existen mezquitas con una pequeña habitación anexa reservada a las mujeres.

Finalmente, en los últimos años han llegado jóvenes procedentes de Ghana y otros países del área subsahariana. Este colectivo está creciendo rápidamente (ahora se presupone que son alrededor de unas trescientas personas).

La mayoría de las personas mayores que viven en Vic son predominantemente trabajadores retirados de la industria, con bajos ingresos, propietarios de un piso en el centro de la ciudad sin ascensor, mayoritariamente nacidos en Vic o Cataluña y como mínimo un hijo o hija. El $62 \%$ reciben ayuda de los parientes que viven con ellos, el $6 \%$ de los parientes que viven en otros lugares y sólo un $1 \%$ disponen de ayuda de los Servicios Sociales (ISPA, 1993).

Hemos estudiado nueve unidades domésticas con diferentes grados de dependencia de los Servicios Sociales. En algunos casos extremos encontramos personas que viven solas en habitaciones dentro del Centro de Día, con todos los servicios necesarios. Son personas dependientes con claras incapacidades. El caso intermedio está formado por personas que usan el Centro de Día como complemento a un soporte familiar primario. Finalmente encontramos personas que son usuarios de servicios puntuales como la telealarma o el cátering $o$, también, personas de clase media que participan en el programa "Viure i Conviurem, un programa que permite a las personas mayores hospedar estudiantes universitarios en sus casas a cambio de compañía. 
Vamos a estudiar de forma breve dos unidades domésticas de la categoría de menor dependencia. Nicolau y Mercè son hermano y hermana (66 y 78 años respectivamente) que viven en un pequeño piso alquilado. Mercè ha trabajado en el servicio doméstico toda su vida y Nicolau trabajaba como panadero. Ambos son solteros. Mercè tiene serios problemas de autonomía pero aún es capaz de hacer algunas pequeñas tareas domésticas. Nicolau, ahora con buena salud, se ocupa básicamente de la compra diaria. Una vez por semana, una persona del Departamento de Bienestar Social acude a ayudarlos en las tareas domésticas. Mercè y Nicolau tienen una hermana más joven, Angelina, en quien ellos confían en casos de crisis, como en el reciente episodio en el que Nicolau estuvo hospitalizado a causa de una enfermedad seria y Angelina se trasladó a Vic hasta que la situación se normalizó (ella vive con sus dos hijos a cinco horas de Vic en transporte público).

En el siguiente gráfico podemos ver la red social alrededor de la unidad doméstica. Esta representación es un escalado multidimensional de una matriz que contiene valores de 4 variables (frecuencia de interacción, intensidad percibida de la relación, canal utilizado para el contacto y tipo de relación). Podemos ver que Angelina está muy próxima a la pareja. También, podemos ver la forma de estrella de la red, relaciones puntuales y aisladas con la excepción de

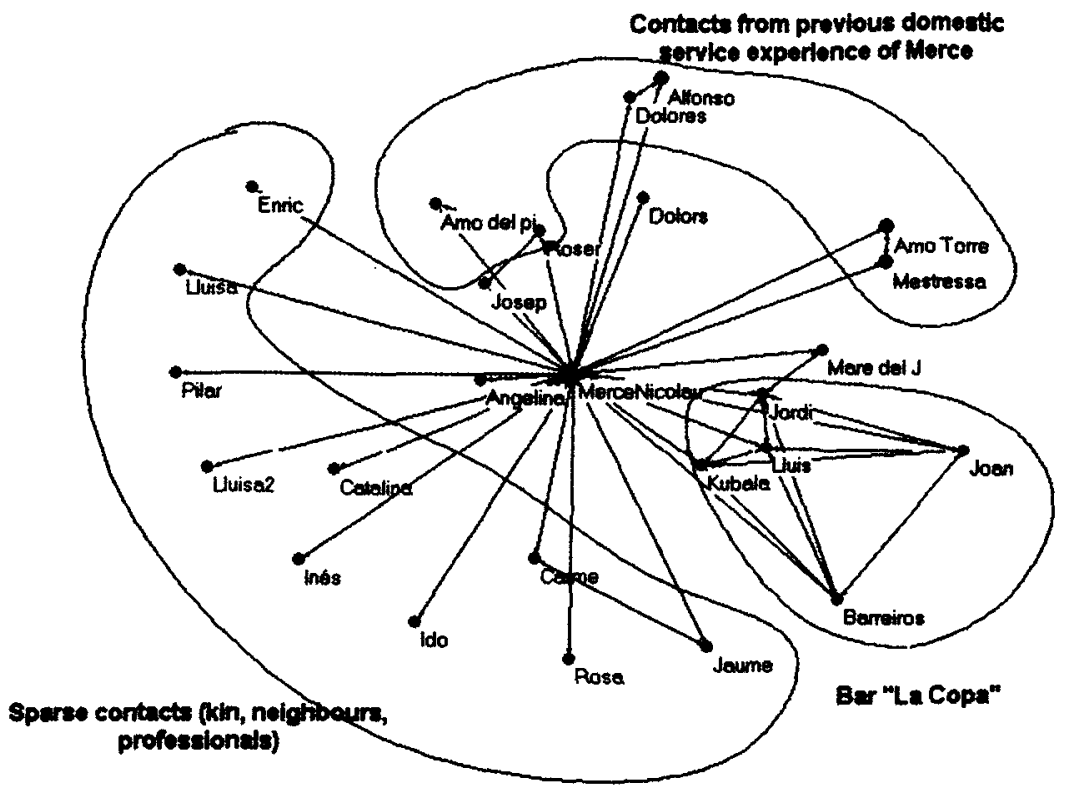


pequeños grupos (relaciones de la experiencia previa de trabajo en el servicio doméstico de Mercè) o el bar "La Copa", un lugar donde Nicolau se encuentra con algunos amigos suyos a diario.

El intercambio social más importante es la socialización (pequeñas charlas, breves visitas, jugar en el bar) con la excepción de una vecina que les ayuda en las tareas domésticas de manera espontánea y el soporte clave de la hermana, que los visita periódicamente.

El segundo caso, Mar y Joan (86 y 92 años respectivamente), está compuesto por dos viudos que recientemente comparten una vida en común. Viven solos en un piso alquilado en la ciudad, pero tienen una fuerte dependencia de ayuda la hija de Mar como puede ser observado en el siguiente gráfico.

El hijo de Joan contribuye con alguna ayuda económica pero la frecuencia del contacto es muy baja. Mar no puede andar sin ayuda y Joan tiene problemas para mantener una conversación a causa de sus problemas de oído. Joan acude al Centro de Día, cerca de su piso, para recoger la comida de la pareja. Cada día va a un local para gente mayor y juega con sus compañeros.

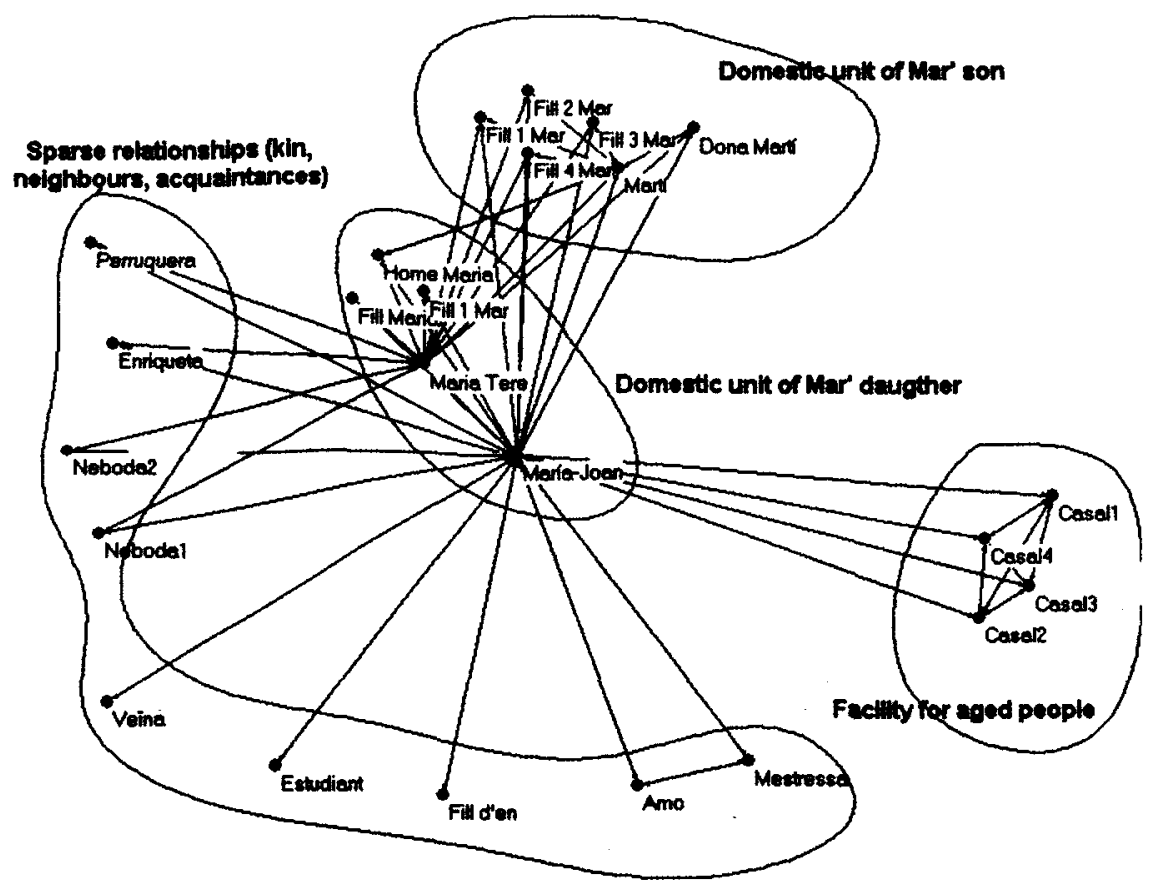


La tipologia de la red es similar a la anterior: los parientes contribuyen en la ayuda doméstica y económica mientras que el resto de los intercambios sociales pueden ser clasificados como socialización.

En el caso del colectivo beréber hemos recogido datos de nueve hogares. Tal como se ha dicho anteriormente, el hogar es básicamente el lugar de las mujeres mientras los hombres trabajan fuera y usan los espacios públicos para encontrarse con otros hombres en bares beréberes de la ciudad, o en bares regentados por beréberes. Estas familias viven en pisos de alquileres baratos en el centro de la ciudad. En el siguiente gráfico podemos ver la estructura de una unidad doméstica (no tenemos información sobre las relaciones de los hombres en el trabajo). Es posible identificar tres grupos principales: parentesco, vecinos beréberes y compañeras beréberes de un curso de aprendizaje del catalán. Es interesante que ambas, vecinas y compañeras de clase se han conocido en Vic por primera vez, pero hay algunos casos en los que se conocen porque en Marruecos vivían en las mismas zonas y pueblos.

Los intercambios sociales entre vecinos beréberes son muy importantes y existe una ayuda financiera regular hacia los familiares que residen en Nador. A medida que la familia residente en Marruecos muere o emigra a Cataluña u otros destinos europeos, estas ayudas económicas son cada vez menos importantes. En el caso de las compañeras de clase, el intercambio social es básicamente la socialización.

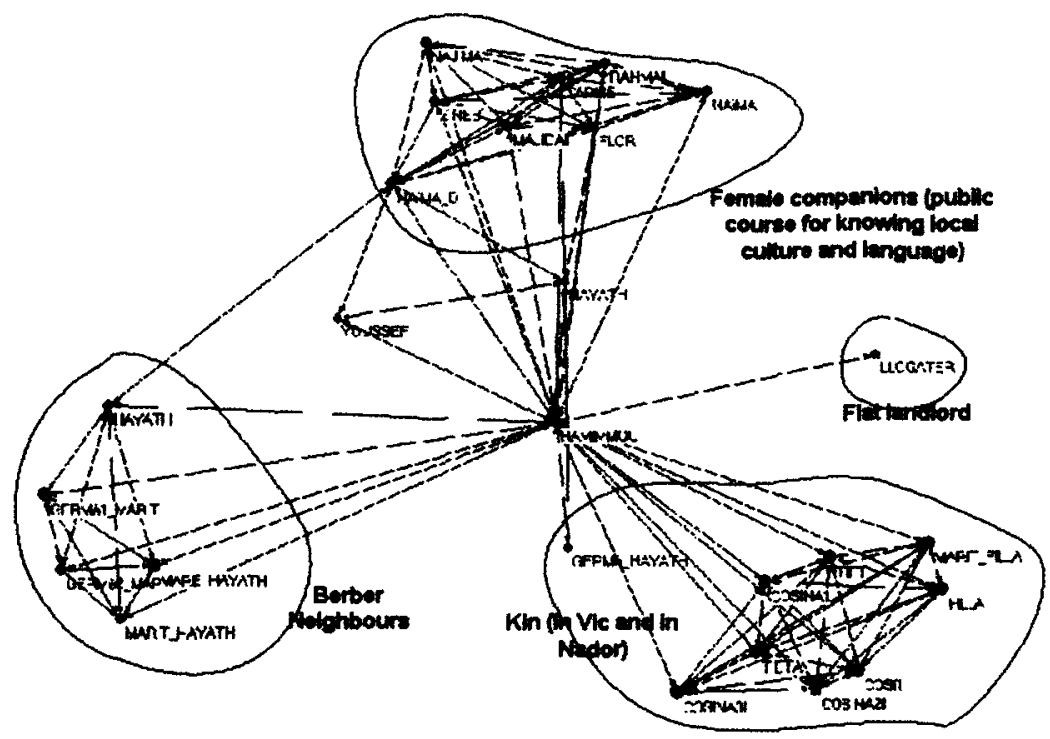


En este gráfico podemos identificar tres grandes grupos a partir de la unidad doméstica de las mujeres de la familia de Hammimou, residente en Vic desde hace dos años a través de la reagrupación familiar. La madre de la familia se queda casi todo en día en casa realizando las tareas domésticas con la ayuda de sus hijas. Solamente habla el idioma beréber y esto le representa un obstáculo para salir a la calle y hacer algunas compras por sí misma. Su mayor contacto es con la familia que reside en Vic y algunos vecinos, sobretodo con una mujer casada, más joven que vive en el mismo bloque, Hayath. Dos de las hijas, Seloua y Soumiyia acuden a clases de catalán, donde han entrado en contacto con otras mujeres beréberes residentes en Vic y con la profesora, Nadia, también de origen beréber. La relación de estas mujeres no va más allá, aparentemente, de estas clases. La menor de las hijas, Samira, acude a las clases que se hacen en el mismo centro al que acuden sus hermanas, dependiente del Ayuntamiento de Vic. A este centro acuden otros chicos y chicas beréberes, pero también jóvenes catalanes con dificultades de aprendizaje. También tiene a Nadia como una de sus profesoras. El padre de Samira trabaja en la construcción y habla bien el castellano. Los tres hijos pequeños acuden a una escuela pública de Vic y hablan bien el catalán, al igual que Samira. Los lazos con los parientes que aún residen en Vic son muy fuertes. Una de las hijas del matrimonio no pudo venir a España porque estaba casada y confían con ilusión que tanto ella como su marido puedan venir en el futuro.

Pese a que nuestros datos son incompletos (tenemos una información muy escasa sobre los contactos e intercambios de los hombres beréberes), podemos analizar los intercambios sociales identificados en las 18 unidades domésticas (ver la siguiente tabla).

\begin{tabular}{lcrcrrr}
\hline Intercambio & Catalán & $\%$ & Beréber & $\%$ & Total & $\%$ \\
\hline Socialización & $\mathbf{3 7}$ & 77 & 45 & 35 & 82 & 46 \\
Trabajo Doméstico & 6 & 13 & 0 & 0 & 6 & 3 \\
Ayuda Económica & 2 & 4 & 3 & 2 & 5 & 3 \\
Indiferenciado & 3 & 6 & 59 & 46 & 62 & 35 \\
Total & 0 & 0 & 22 & 17 & 22 & 12 \\
Tota & 48 & 100 & 129 & 100 & 177 & 100 \\
\hline
\end{tabular}

El rasgo que destaca son los intercambios no especializados entre las unidades domésticas beréberes, de manera que difícilmente es posible diferenciar una unidad doméstica de la otra (parientes y vecinos). Posiblemente esta característica está asociada con los primeros estadios del proceso de inmigración, con solamente un contacto catalán en la red (jel casero!). Posiblemente esta situación 
cambiará en un futuro próximo hacia unas relaciones más especializadas. La ayuda económica tiene los mismos porcentajes en ambos casos (2\%) con la diferencia que las unidades domésticas beréberes que reciben ayuda están en Nador. Esta ayuda es especialmente prominente cuando los inmigrantes regresan a Marruecos por vacaciones en verano, con abundantes regalos para parientes, vecinos próximos y conocidos. Las unidades domésticas tienen que hacer un gran esfuerzo económico para poder mantener su prestigio. Los regalos de productos occidentales son correspondidos por productos típicos locales.

\section{La red de las unidades domésticas}

Las unidades domésticas no están aisladas, incluso en los casos más dependientes. Éstas están conectadas de diferentes formas. En el siguiente gráfico podemos ver la red de las unidades domésticas y los Servicios Públicos u ONG relacionadas (representadas con nodos de mayor tamaño).

Con la excepción de Nasredine (beréber), el resto de las personas inmigrantes están sujetas a una única institución pública. Naturalmente, la cadena de contactos con las organizaciones públicas no está limitada al Departamento de Bienestar Social y sus locales e instalaciones, sino que se extienden a los servicios públicos de salud y educación.

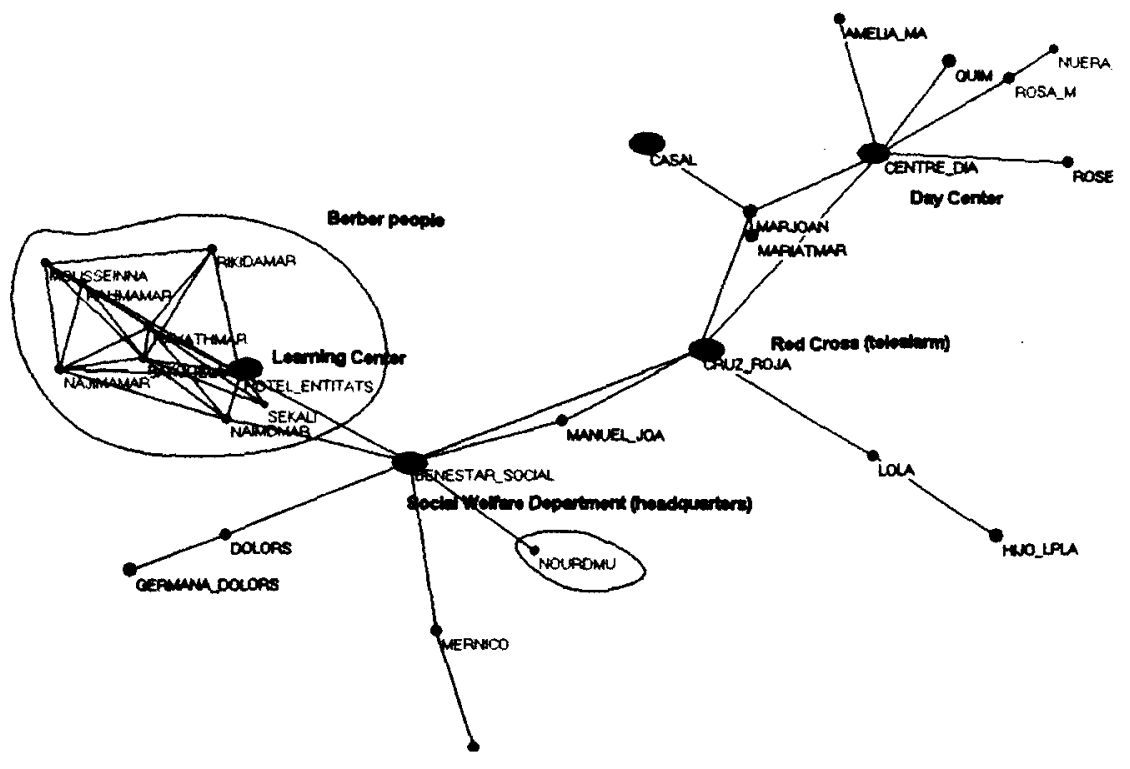


Además, la segregación de los beréberes puede ser explicada porque son más jóvenes, las mujeres trabajan en casa y cuidan ellas mismas de las personas mayores. No obstante, la red de las unidades domésticas y las organizaciones públicas nos permite apreciar el rol que desempeñan. Las decisiones sobre la organización de nuevos servicios, los cambios en la organización de la estructura de los servicios públicos no son solamente cuestiones técnias o decisiones neutrales sino que influencian de manera decisiva en la red de relaciones de las unidades domésticas. De hecho, el mismo fenómeno que podemos observar en el seno de las organizaciones se reproduce a un nivel más alto, a saber, que los diseños organizativos determinan el rango de los contactos informales que podemos encontrar en una organización (Molina, 2001).

Reciprocidad fuera de la esfera doméstica

Tal como hemos dicho anteriormente, los intercambios sociales que se dan fuera de la esfera doméstica están formalmente restringidos a las normas de etiqueta, confiando en el precio o la norma para los intercambios económicos o burocráticos. Naturalmente, el proceso de socialización no está prohibido y es gracias a esta socialización que podemos hablar del fenómeno del capital social. En relación con nuestra investigación podemos encontrar diversos ejemplos de reciprocidad fuera de la esfera doméstica que vamos a explicar brevemente.

En primer lugar, la implicación personal de los profesores del centro de enseñanza con los beréberes (esfera del dominio público). Esta implicación se da más allá de sus obligaciones burocráticas e incluye celebraciones (como el fin del Ramadán), soporte emocional, información acerca de ofertas de trabajo, etc. La figura de Nadia, que habla catalán y beréber, aparte del árabe marroquí, es muy importante para ambas comunidades porque ella es el "puente" entre las dos culturas.

En segundo lugar, el caso de Nasredine, una de las personalidades más importantes de la comunidad beréber. Regenta una carnicería halal (un lugar donde la carne se obtiene de animales que han sido sacrificados según el ritual islámico y en la que se pueden encontrar otros productos propios de uso común de la comunidad marroquí), un bazar y un bar beréber. Su éxito es celebrado tanto en Nador como en Vic. Amigos próximos y hermanos más jóvenes trabajan para él. El prestigio de Nasredine es mantenido a través contribuciones al- 
truistas a personas y familias, como la acogida de emigrantes con dificultades que llegan a Vic solamente con su nombre en el bolsillo dándoles comida hasta que lo necesiten, o financiando el coste de la repatriación de los difuntos para su entierro en Nador, en suelo musulmán. Estos intercambios, que tienen lugar en la esfera del mercado, pueden ser explicados por el llamado «dilema del comerciante" (Evers y Schrader, 1994), esto es, la necesidad de distanciarse socialmente de los clientes para hacer funcionar el negocio implica un rechazo de la comunidad. Para solventar este dilema hay diversas opciones que pueden incluir especializaciones étnicas o la inmigración de minorías comerciantes. En nuestro caso, la opción puede ser la adquisición de prestigio a través de gastos sociales, lo cual permite superar este rechazo, o, al menos, amortiguarlo.

En tercer lugar, las donaciones o intercambios caritativos. Los jóvenes negros y algunas personas del Magreb en situaciones difíciles son atendidas por los monjes católicos o, en otros casos, por las redes de ONGs con roles especializados (asistencia legal, refugio, etc.). El caso de los monjes es muy especial porque ellos dejan que los jóvenes negros celebren sus rituales no católicos en los locales de la iglesia. Sin embargo, pensamos que este intercambio puede ser interpretado como burocrático o basado en la norma, en la esfera del dominio público. El comportamiento de las organizaciones puede explicarse mejor sin tomar en consideración las motivaciones de sus miembros (voluntarios de ONGs, o padres claretianos de la Iglesia; ver Molina, 1997). Las donaciones en el caso de la caridad islámica (uno de los cinco pilares del Islam) están organizados por las unidades domésticas a través de la donación de limosna a personas conocidas que se encuentran en dificultades. En ambos casos (organizaciones religiosas y unidades domésticas musulmanas) podemos encontrar la forma extrema del regalo, que es, el "regalo puro" o el presente sin retorno (ver Laidlaw, 2000).

\section{Conclusión}

A través del análisis de las unidades domésticas de los dos colectivos en Vic sugerimos algunas ideas para valorar el papel de la reciprocidad en una sociedad compleja.

En primer lugar, es útil distinguir entre intercambios sociales, a saber, intercambios que crean una obligación de devolver de forma no definida en el 
futuro, e intercambios económicos o burocráticos, formalmente equilibrados y determinados. Estos intercambios sociales se desarrollan naturalmente en la esfera doméstica mientras que están formalmente restringidas a la etiqueta en las esferas del mercado y la administración pública. También sugerimos que los estudios de capital social expresan el fracaso de esta restricción.

En segundo lugar, les intercambios sociales entre unidades domésticas pueden ser categorizados como socialización, trabajo doméstico y ayuda financiera. Hemos visto que los vecinos y los conocidos proveen el primero y el parentesco el resto (excepto en el caso de los vecinos beréberes).

En tercer lugar, la red de unidades domésticas y organizaciones públicas y privadas puede ser una perspectiva útil para entender mejor la estructura social y su dinámica.

\section{Bibliografía}

ADLER-LOMNITZ, Larissa (1994): Redes sociales, cultura y poder. Ensayos de antropologia latinoamericana, México: Miguel Angel Porrúa.

AIXELA, Yolanda (2000): Mujeres en Marruecos. Un análisis desde el parentesco y el género, Ed. Alborán, Ed. Bellaterra, Barcelona.

AJUNTAMENT DE VIC (2000): Fòrum Intercultural de Vic. Cicle de Xerrades sobre Fenomen Migratori i Procés d'Adaptació 97-99, Vic, Servei de Publicacions.

ATtias-DONFUNT, Claudine; Rozenkier, Alain (1996): "The Lineage-Structured Social Networks of Older People in Francem, en LITWIN, Howard (ed.) (1996): The Social Networks of Older People: A Cross-National Analysis (32-53).

BlaU, Peter M (1964): Exchange and Power in Social Life, New York: John Wiley.

CAMPS, Gabriel (1998): Los beréberes. De la orilla del Meditarráneo al límite meridional del Sáhara, Ed. CIDOB, Ed. Icària, Barcelona.

Colectivo Ió (1994): Marroquins a Catalunya, Col-lecció Mare Nostrum, Enciclopedia Catalana, Barcelona.

EVERS, Hans-Dieter; SCHRADER, Heiko (1994): The Moral economy of trade ethnicity and developing markets, London: Routledge.

GouldNeR, Alvin W. (1960): "The Norm of Reciprocity: A Preliminary Statement», American Sociological Review, 25 (161-178). 
Granovetter, Mark S. (1985): «Economic Actions and Social Structure: The Problem of Embeddedness", American Journal of Sociology (481-510).

HOMANS, George (1958): "Social behaviour as exchange", American Journal of Socio$\log y, 62,(597-606)$.

ISPA (1993): La gent gran de Vic. Anàlisi sociologica de les característiques $i$ condicions de vida de la població, Vic, Ajuntament de Vic.

KAPFERER, Bruce (1972): Strategy and transaction in an African factory: African workers and Indian management in a Zambian town, Manchester: Manchester University Press.

LAIDLAW, James (2000): "A Free Gift Makes No Friends", Royal Anthropological Institute, 6 (617-634).

LiN, Nan (2001): Social Capital: $A$ Theory of Social Structure and Action, Cambridge: Cambridge University Press.

MALINOWSKI, B. (1986): Els argonautes del Pacific Occidental. [Argonautes of the Western Pacific. An Account of Native Enterprise and Adventure in the Archipelagoes of Melanesian New Guinea, 1922], Barcelona: Edicions 62.

- (1977): El cultivo de la tierra y los ritos agricolas en las islas Tobriand. [Coral Gardens and their Magic. Soil-tilling and agricultural rites in the Tobriand Islands, 1935], Barcelona: Labor Universitaria.

MAUSS, Marcel (1979): «Ensayo sobre los dones, motivo y forma del cambio en las sociedades primitivas [Essai sur le don, 1925]", en Sociologia y antropologia, Madrid: Tecnos.

MogA, Vicente (ed.) (1992): Revista Aldaba: Amazigh - Tamazight. Debate Abierto, Servicio de Publicaciones del Centro Uned, Melilla.

MOGA, Vicente; AHMED, Rachid (eds.) (1998): Mujer tamazight y fronteras culturales, Servicio de Publicaciones del Centro Uned, Melilla.

MolinA, José Luis (1997): «La solidaritat avui: el cas d'una ONG pel desenvolupament», Revista d'Etnologia de Catalunya, 11 (78-85).

- (2001): "The informal organizational chart in organizations», Connections, vol. 14, Spring 2001 (78-91).

MORENO, Paz, y NAROTZKY, Susana (2000): «La reciprocidad olvidada: reciprocidad negativa, moralidad y reproducción social», en Hispania, Consejo Superior de Investigaciones Cientificas.

POLANY, Karl (1957): «The economy as instituted process", in POLANY, K.; ARENSBERG, C.W., and PeARson, H.W. (eds.): Trade and Market in the Early Empires, New York: Free Press. 
- (1992): La gran transformación. Los origenes politicos y económicos de nuestro tiempo [The Great Transformation. The Political and Economic Origins of our Time, 1944], México: FCE.

RIFKIN, Jeremy (1996): El fin del trabajo. Nuevas tecnologias contra puestos de trabajo:el nacimiento de una nueva era [The end of work. The decline of the global labor force and the dawn of the post-market era, 1994], Barcelona: Paidós.

- (2000): La era del acceso. La revolución de la nueva economia [The Age of Access, 2000], Barcelona: Paidós.

SAHLINS, Marshall (1977): Las sociedades tribales [Tribesmen], Labor, Barcelona.

SEgura I MAS, Antoni (1994): El Magreb: del colonialismo al islamismo, Publicacions de la Universitat de Barcelona, Barcelona.

TerRadAs, Ignasi (2000): «Reciprocidad ¿para qué?», Proyecto PB98-1238, Programa Sectorial de Promoción General del Conocimiento.

VALCÁRCEL GUITIAN, Rosario (1999): Etnografia de les families immigrants marroquines, tesina, Master en Antropologia Aplicada, Universitat Autònoma de Barcelona. 